Voix et Images

volxetimages

\title{
Du Canada français au Québec libre de Jean-Claude Robert en passant par Nationalismes et politique au Québec de Léon Dion
}

\section{André Vanasse}

Volume 1, numéro 2, décembre 1975

Raoul Duguay

URI : https://id.erudit.org/iderudit/290083ar

DOI : https://doi.org/10.7202/290083ar

Aller au sommaire du numéro

Éditeur(s)

Les Presses de l'Université du Québec

ISSN

0318-9201 (imprimé)

1705-933X (numérique)

Découvrir la revue

Citer ce compte rendu

Vanasse, A. (1975). Compte rendu de [ Du Canada français au Québec libre de Jean-Claude Robert en passant par Nationalismes et politique au Québec de Léon Dion]. Voix et Images, 1(2), 290-295. https://doi.org/10.7202/290083ar d'utilisation que vous pouvez consulter en ligne. 


\section{Du Canada français au Québec libre de Jean-Claude Robert en passant par Nationalismes et politique au Québec de Léon Dion}

Au moment d'écrire ce texte, Pierre Elliott Trudeau vient tout juste de faire son mémorable discours où il consacre de façon non équivoque son "libéralisme économique» en obligeant les travailleurs, et eux seuls, à payer le prix de l'inflation. À ce jour, aucune déclaration officielle de Robert Bourassa sinon quelques commentaires soutirés par les journalistes selon lesquels la province de Québec sera la première à respecter les nouvelles lois de M. Trudeau. «Plus ça change plus c'est pareil " (p. 238) répète après tant d'autres Jean-Claude Robert et on ne peut qu'éprouver avec agacement, pour ne pas dire avec désespoir, ce sentiment qu'au Québec «rien 
ne doit changer"! Devant l'attitude de Bourassa comment ne pas faire le lien avec celle de la plupart des autres premiers ministres du Québec? A titre d'exemple, cette réflexion de J.-C. Robert à propos du gouvernement de L.-A. Taschereau: «Devant la hausse considérable du chômage, le ministre réagit mollement (mon Dieu, est-ce possible?); les initiatives viennent du gouvernement fédéral auquel il emboîte le pas." (p. 178)

En vérité je me demande ce que signifie le titre du livre de JeanClaude Robert: Du Canada français au Québec libre, histoire d'un mouvement indépendantiste ${ }^{\prime}$. Ce volume, de plus de trois cents pages, est le plus démoralisant qu'il m'ait été donné de lire. Il l'est d'autant plus que la description et l'analyse que fait J.-C. Robert de notre situation historique depuis 1760 jusqu'à nos jours est fort pertinente. Le travail est clair, instructif et même si l'auteur répète des faits connus depuis longtemps, il nous renseigne par contre sur beaucoup d'autres aspects qui l'étaient moins.

Mais comment ne pas être atterré devant ce très sérieux bilan. De 1760 à 1975 que se passe-t-il en ce qui concerne l'indépendance du Québec? À peu près rien si ce n'est deux événements majeurs à savoir la période de 1815 à 1837 qui aboutit à la révolte de 1837 rapidement matée et la période de 1960 à 1966 appelée, pour notre propre satisfaction narcissique, «révolution tranquille» et qui n'est, en fait, qu'un simple et très bref rattrapage par rapport à l'ensemble du pays dénommé, comme on le sait trop bien, Canada.

On peut toujours rappeler, comme le fait si bien Jean-Claude Robert, les remous causés ou par l'Affaire Riel, ou par le triste sort réservé aux écoles françaises du Manitoba et celles du Nouveau-Brunswick (ce que les "Canadians» peuvent faire n'est évidemment pas permis aux Québécois!) ou par les deux conscriptions ou par quelques menus faits divers, mais la masse des événements est telle que je ne peux que constater l'échec d'une population qui, soumise à des pressions économiques, politiques et culturelles imposées plus ou moins violemment par le groupe dominateur, est incapable de se donner les moyens de prendre en mains "le gouvernail de sa destinée".

A lire le texte de J.-C. Robert je me dis que Lord Durham était vraiment un homme intelligent et je me demande comment il se fait que ses projections ne se soient pas encore réalisées. (Durham, entre autres choses, était persuadé «que les Français une fois placés en minorité, par suite du cours naturel et légitime des événements et par le fonctionnement des causes naturelles abandonneraient leurs vaines espérances de nationalité... La perte irrémédiable de tout espoir de succès atténuerait peu à peu les haines actuelles et porterait graduellement les Canadiens français à accepter leur nouveau statut politique». p. 123)

1. Du Canada françaıs au Québec llbre, historre d'un mouvement indépendantiste, Paris, Flammarion, 1975, 315 pages. 
D'une certaine façon le fait français en Amérique tient du miracle (pour reprendre le vocabulaire théocentrique qui fut longtemps le nôtre!) mais ce miracle risque de ne plus se renouveler. Car il faut admettre que la situation a beaucoup évolué depuis les débuts de la colonie et que les conditions précaires qui nous avaient favorisés (majorité écrasante des francophones dans la "province of Québec", nécessité, pour l'Angleterre, de procéder avec prudence face à la menace réelle et constante des États-Unis etc., etc.) n'existent plus depuis longtemps (est-il nécessaire de rappeler que cette "province of Québec" après avoir été divisée en deux parties celle du Bas et du Haut-Canada, est devenue ensuite cet immense pays dont huit, pour ne pas dire neuf, des dix provinces sont majoritairement anglophones).

La société québécoise (elle s'appelait autrefois canadienne-française, ne l'oublions pas!) glisse lentement vers sa disparition si elle ne prend pas les moyens qui s'imposent. Comment y parviendra-t-elle, elle qui a accepté toutes les humiliations, tous les compromis, desservie en cela par un clergé prêt à toutes les concessions pour maintenir son "pouvoir spirituel" et par une députation malheureusement hypnotisée et terrorisée par le pouvoir anglais? Comment ne pas voir qu'avec la naissance de la Confédération (qui n'était en somme qu'un immense chemin de fer et la possibilité pour beaucoup d'anglophones de faire fortune) "jamais plus les Canadiens français ne pourront s'opposer avec force et faire échouer les politiques de leurs adversaires $»$. (p. 142)

Au contraire, plus le temps avance et plus la disproportion augmente entre les anglophones et les francophones. Le Québec doit non seulement livrer un semblant de lutte contre cette énorme machine fédérale mais se trouve débordé à l'intérieur même de ses propres frontières (inutile d'insister sur le problème scolaire de Saint-Léonard).

Que reste-t-il à faire sinon d'espérer un miracle (toujours ce langage théocentrique) qui ne se réalisera probablement jamais! Voilà les conclusions auxquelles j'arrive après avoir lu le livre de Jean-Claude Robert. Conclusions démoralisantes et accentuées encore plus après la lecture de Nationalismes et politique au Québec ${ }^{2}$ de Léon Dion. Dans ce livre on y tient les mêmes propos mais dans un langage différent et un peu plus sophistiqué. Qu'est-ce que cela peut bien me faire si Léon Dion affirme que le mouvement de réforme entrepris par le gouvernement Lesage n'est pas né ex nihilo mais que l'idéologie libérale qui soutenait le nouveau gouvernement au pouvoir avait été d'abord un phénomène souterrain décelable à plusieurs niveaux? J'accepte fort bien que, pour des besoins théoriques et par souci d'objectivité, Léon Dion prétende que l'idéologie libérale «soustendit la plupart des nombreux courants d'opposition extra-parlementaire,

2. Léon Dion, Nationalismes et politique au Québec, Montréal, Hurtubise-HMH, 1975,177 pages. 
depuis la grève d'Asbestos jusqu'à celle de Louiseville et depuis le mouvement suscité par la Faculté des sciences sociales de Laval à l'instigation de son Doyen, le père Georges Henri Lévesque, jusqu'à la victoire libérale du 22 juin $1960 »$. (p. 58) Eh bien, soit! mais que puis-je dire quand le même Léon Dion, après avoir fait ces nécessaires mises au point, constate que les têtes d'affiche qui furent à l'origine de l'idéologie libérale des années 60 se sont soudainement envolées vers la scène fédérale, sous le gracieux vocable des "trois colombes", et que «le malheur est [...] que par les postes de premier plan qu'ils occupent sur la scène politique ils sont maintenant en mesure d'imposer leurs vues". (p. 59) Mêmes commentaires aussi pour le Père Georges-Henri Lévesque qui, débordé par les forces montantes de sa propre faculté, basculait lui aussi dans le plat d'argent outaouais.

Et Dion de conclure laconiquement: “Tant d'argent et d'énergies investis sans que ne soit attenué l'état de dépendance du Québec par rapport au Canada anglais et aux États-Unis - le contrôle de l'économie québécoise est aujourd'hui encore autant entre les mains des "étrangers" qu'il l'était en 1960 et sans que la position des Canadiens français par rapport à celle des autres groupes ethniques dans l'échelle des revenus ne tende à s'améliorer. » (p. 79)

Somme toute, le seul régime libéral du Québec depuis la Confédération n'a pas donné les résultats escomptés. Peut-être les dés étaient-ils pipés d'avance? "S'il n'en avait tenu qu'au Premier Ministre Jean Lesage au cours de la première année de son administration surtout, il y a même lieu de penser qu'il se serait avec soulagement laissé retomber dans le silIon familier du nationalisme conservateur. " (p. 75)

Évidemment, pour quelques naïfs il reste encore le Parti québécois mais les craintes que plusieurs ont ressenties à son égard, à mesure que le temps avançait, sont clairement exprimées par Léon Dion: «Mais le Parti québécois ne pourra remplir la difficile mission politique que nombre de ceux qui le supportent lui confient que si, lui-même, réussit à devenir un parti populaire, démocratique au sommet et à la base, et s'il parvient à surmonter la tentation technocratique à laquelle il paraît bien près de succomber. S'il devait tomber dans la trappe technocratique qui le guette, il ne serait plus, au mieux, qu'un autre parti libéral et, au pire, qu'un parti "techno-démagogique" selon l'expression que j'ai suggérée dans Société et politique: la vie des groupes. L'accession du Parti québécois comme force politique significative au Québec ne représente pas la fin de la lutte pour la promotion nationale et sociale. Elle est toutefois un signal irrécusable, pour les protagonistes du nationalisme social-démocrate comme pour ceux du nationalisme socialiste, que le moment est venu de se réveiller et de passer à l'action. Si l'instrument ne convient pas à la mission qu'il lui incombe d'accomplir, il est grand temps de le réparer ou d'en forger un autre. " (p. 126-127) 
Or, face à l'effritement constant de l'actuel Parti libéral, on peut penser que le Parti québécois a certaines chances de parvenir au pouvoir. Mais la position ferme, concernant le référendum, exprimée lors de la dernière rencontre à Victoriaville ("oui nous acceptons la formule Morin ") me laisse présager le pire. J'ai nettement l'impression que le Parti québécois, s'il accédait au pouvoir, répéterait les impertinences de Duplessis face au pouvoir fédéral (nous savons le prix qu'il a fallu payer, particulièrement en ce qui concerne les universités). Évidemment nous changerions d'idéologie: de l'agriculturisme nous passerions au mythe technologique mais le résultat serait le même. Car peut-on penser que cette frayeur extrême de la réelle prise du pouvoir puisse aboutir à l'indépendance? La question du référendum est en soi piégée. Elle présuppose le miracle de la conversion auquel je ne crois pas. Pourquoi donner à la population le choix de dire oui (lors des élections) pour ensuite lui permettre de dire "non" sinon parce que le Parti québécois enrage de ne pas être au pouvoir!

Ce qui me fascine dans ce jeu tactique, ce ne sont pas ses résultats possibles sinon probables mais ce qu'il camoufle. Je me dis que la peur de l'autorité doit être solidement ancrée en nous pour nous obliger à procéder de façon aussi louvoyante. Si la psychanalyse a parlé du traumatisme de la naissance on pourrait, en termes socio-psychanalytiques applicables au Québec, parler du traumatisme de la conquête, à la suite de Ferron et de d'autres.

Car il semble y avoir au Québec un pattern de comportement inconscient qui oblige les classes dirigeantes et, partant, les populations qui les suivent à proposer sous le couvert de la rationalité (entendre: efficacité électorale) des solutions contradictoires et symptômatiques. A ce titre la solution proposée par le Parti québécois répond parfaitement à cette description. Elle présuppose que la société québécoise puisse à la fois répondre oui et non en toute bonne foi. En termes descriptifs on pourrait illustrer le pattern de la façon suivante: un père ordonne à son fils de se laver les mains. L'enfant, en se lavant consciencieusement les mains, répète à voix haute: «Non, je ne me laverai pas les mains... Non, je ne me laverai pas les mains..."

Cet exemple n'est pas aussi simpliste qu'on voudrait le croire. II explique par exemple l'attitude des Québécois face aux mesures de guerre en 1970, c'est-à-dire une acceptation et une révolte contre des mesures jugées iniques (inutile de répéter la sempiternelle phrase: “Face à la menace du F.L.Q. le ministre réagit mollement; les initiatives viennent du gouvernement fédéral auquel il emboîte le pas"). La meilleure façon d'expliquer le phénomène c'est de bien comprendre le sentiment ambivalent des Québécois devant le dominateur. L'Anglais n'est-il pas celui qui a conquis et permis la survie (le problème était encore plus déchirant lors de l'application des mesures de guerre puisque le premier ministre qui les appliquait était à la fois le représentant du pouvoir anglais et simultanément un des nôtres). 
C'est à partir de cette problématique qu'on peut comprendre la compulsion de répétition du peuple québécois (ou de ses représentants). Déchiré entre deux sentiments il cherche constamment une impossible et absurde synthèse, celle de contester et de ne pas rejeter le dominateur. A ce titre, le Parti québécois tombe dans la même trappe que tous les autres partis que nous avons connus jusqu'à maintenant.

La situation me paraît finalement tragique puisque à côté du Parti québécois, défini par Dion comme le représentant de l'idéologie socialedémocrate mais dont le risque de plus en plus perceptible est de tomber dans l'idéologie nationaliste libérale ou technocratique, il ne reste plus que les tenants du nationalisme socialiste qui, globalement, n'ont rien de nationaliste. En fait, ces groupements marginaux obnubilés qu'ils sont par le mythe «internationaliste" évacuent presque systématiquement le problème épineux de la culture et de la langue pour concentrer leurs énergies sur la lutte des classes et la guerre ouverte contre le système capitaliste ${ }^{3}$. Or, à mon avis, les tenants de cette idéologie, parce qu'ils brûlent les étapes (le peuple québécois doit d'abord se donner une identité et une certaine autonomie) sont appelés à jouer un rôle marginal (même s'il est utile) dans la conjoncture actuelle.

Car pour l'instant la force montante est bien celle du Parti québécois et advenant le cas d'une victoire (très hypothétique, il faut bien l'admettre) aux prochaines élections, je redoute qu'il faille répéter après Jean-Claude Robert, Léon Dion et beaucoup d'autres: "Plus ça change, plus c'est pareil. » 